\title{
BMJ Open Haemorrhage rates of ruptured and unruptured brain arteriovenous malformation after radiosurgery: a nationwide population-based cohort study
}

\author{
Cien-Leong Chye, ${ }^{1,2}$ Kuo-Wei Wang, ${ }^{1,3}$ Han-Jung Chen, ${ }^{1,2}$ Shyh-An Yeh, ${ }^{1,4}$ \\ James Taoqian Tang, ${ }^{1,5}$ Cheng-Loong Liang (D) 1,2
}

To cite: Chye C-L, Wang K-W, Chen H-J, et al. Haemorrhage rates of ruptured and unruptured brain arteriovenous malformation after radiosurgery: a nationwide populationbased cohort study. BMJ Open 2020;10:e036606. doi:10.1136/ bmjopen-2019-036606

- Prepublication history for this paper is available online. To view these files, please visit the journal online (http://dx.doi. org/10.1136/bmjopen-2019036606).

Received 21 December 2019 Revised 22 June 2020 Accepted 26 August 2020

D) Check for updates

(c) Author(s) (or their employer(s)) 2020. Re-use permitted under CC BY-NC. No commercial re-use. See rights and permissions. Published by BMJ.

${ }^{1}$ I-Shou University, School of Medicine, Kaohsiung, Taiwan ${ }^{2}$ Department of Neurosurgery, EDa Hospital, Kaohsiung, Taiwan ${ }^{3}$ Department of Neurosurgery, EDa Cancer Hospital, Kaohsiung,

Taiwan

${ }^{4}$ Department of Radiation Oncology, E-Da Hospital, Kaohsiung, Taiwan

${ }^{5}$ Department of Community Medicine, E-Da Hospital, Kaohsiung, Taiwan

Correspondence to Cheng-Loong Liang; ed100183@edah.org.tw

\section{ABSTRACT}

Objectives The present nationwide population-based cohort study aims to assess the effectiveness of gamma knife radiosurgery (GKS) on ruptured and unruptured brain arteriovenous malformations (AVMs) by evaluating the haemorrhage rates.

Design A nationwide, retrospective cohort study.

Setting Taiwan National Health Insurance Research Database (NHIRD).

Participants An observational study of 1515 patients who were diagnosed with brain AVMs between 1997 and 2013 from the Taiwan NHIRD.

Primary outcome and secondary outcome measures We performed a survival analysis using the Kaplan-Meier method. Multivariate Cox proportional hazards regression models were used to explore the relationship between treatment modalities (GKS vs nonGKS) and haemorrhage, adjusted for age and sex.

Results The GKS and non-GKS groups included 317 and 1198 patients, respectively. Patients in the GKS group (mean \pm SD, 33.08 \pm 15.48 years of age) tended to be younger than those in the non-GKS group $(37.40 \pm 17.62)$ $(p<0.001)$. The 15-year follow-up revealed that the rate of bleeding risk was lower in the GKS group than in the non-GKRS group (adjusted HR (aHR) $0.61 ; 95 \% \mathrm{Cl} 0.40$ to 0.92). The bleeding risk of ruptured AVMs was significantly lower in GKS group than in the non-GKS group (aHR 0.34; $95 \% \mathrm{Cl} 0.19$ to 0.62 ). On the other hand, the bleeding risk of unruptured AVMs was higher in the GKS group than in the non-GKS group (aHR 1.95; 95\% Cl 1.04 to 3.65). In the unruptured AVM group, the incidence of bleeding was significantly higher among patients in the GKS group that were of $>40$ years of age (aHR $3.21 ; 95 \% \mathrm{Cl} 1.12$ to 9.14 ). Conclusions GKS is safe and it reduces the risk of haemorrhage in patients with ruptured AVMs. The administration of GKS to patients with unruptured AVMs who are above the age of 40 years old male might increase the risk of haemorrhage.

\section{INTRODUCTION}

Brain arteriovenous malformations (AVMs) are rare, congenital vascular lesions with an incidence and prevalence of approximately
Strengths and limitations of this study

- This is the first nationwide Asian population-based study that evaluated the effect of gamma knife radiosurgery (GKS) on cerebral arteriovenous malformations (AVMs) with a follow-up of 15 years.

- GKS provided an acceptable risk and benefit profile in reducing the subsequent risk of haemorrhage in patients with ruptured AVMs.

- This is a retrospective, population-based study with limited clinical information which detailed clinical and functional outcomes are unavailable.

- Grading of AVMs and neurological severity of the haemorrhage could not be ascertained, which limited our insight into the significance of the effect of GKS on AVMs of different grades.

1 in 100000 and 18 in 100000 , respectively. ${ }^{1}$ According to the largest single-centre and population-based series of AVM cases, haemorrhage is consistently described as the most common initially presenting feature of AVMs, occurring in approximately $50 \%$ of cases at first diagnosis. ${ }^{23}$ While Tong et at demonstrated that haemorrhagic presentation progressively decreases from the first decade of life to the seventh, their findings indicate that patients with AVMs are subject to a lifelong risk of haemorrhage. The rate of haemorrhage is the highest among patients below the age of 30 years, extremely high in children, and significantly lower in patients aged $30-59$ years.

Prevention of intracranial haemorrhage is important for the long-term outcome of AVM intervention. Entailing no significant immediate treatment risk, radiosurgery is the least invasive of the various types of AVM interventions; both the beneficial and adverse effects of radiosurgery are delayed, occurring over 
the course of months to years. ${ }^{5}$ However, until total obliteration of AVM is achieved, radiosurgery-treated AVMs remain exposed to the risk of rupture.

Whether the latency period between radiosurgery and obliteration or AVM's natural history entails a greater risk of haemorrhage is a topic of debate. Maruyama et at analysed the AVM radiosurgery outcomes of a cohort of 500 patients and found that radiosurgery significantly reduced the risk of AVM haemorrhage and the haemorrhage risk of ruptured AVMs to a significantly greater extent than unruptured AVMs; the annual postradiosurgery haemorrhage rates of ruptured AVMs at 1, 2, 3 and $>3$ years were $6.3 \%, 6.8 \%, 6.4 \%$ and $6.3 \%$, respectively. According to a randomised trial of unruptured brain AVMs (ARUBA), the effect of radiosurgery on unruptured AVMs is not beneficial, and the conservative management of patients with unruptured AVMs results in better outcomes than intervention. ${ }^{7}$ However, the study was limited by a short follow-up period and probable selection bias of participants. ${ }^{8}$ The aim of this population-based, case-control study conducted using the Taiwan National Health Insurance Research Database (NHIRD) was to investigate the long-term haemorrhage risk of patients with either ruptured or unruptured AVMs, and the role of gamma knife radiosurgery (GKS) on the risk of bleeding.

\section{MATERIALS AND METHODS}

\section{Study design and sample selection}

Established in 1995, Taiwan's state-run National Health Insurance (NHI) programme covers more than $99 \%$ of the nation's population and has contracts with $97 \%$ of the hospitals in Taiwan. Data analysed in this study were retrieved from the Taiwan NHIRD, which is managed by the Taiwan National Health Research Institute (NHRI). The details of this population-based database have been described previously. ${ }^{9}$ Diagnoses were coded with the International Codes of Disease ninth Edition Clinical Modification (ICD-9-CM).

Individuals with newly defined AVMs having been identified between 1997 and 2013 were retrieved from the NHIRD. The accuracy of AVM diagnoses (ICD-9-CM code 747.81) was confirmed by the inclusion of the corresponding cases in a subdatabase of the NHIRD, the Registry for Catastrophic Illness Patient Database (RCIPD): confirmation of AVM by the review of brain CT, MRI and/or angiography by the members in a specific committee of the NHI is required for patients to be registered in the RCIPD.

A total of 1844 patients were diagnosed with AVM, 329 of whom were excluded on account of having received either surgery and/or embolisation; 1515 patients were thus included in the present study. While GKS (based on the procedure code 37029B) or conservative treatment (non-GKS) could be used at the discretion of the neurosurgeon and radiation oncologist, the decision was contingent on the patient's agreement. Whether or not GKS was administered is documented in the patients'

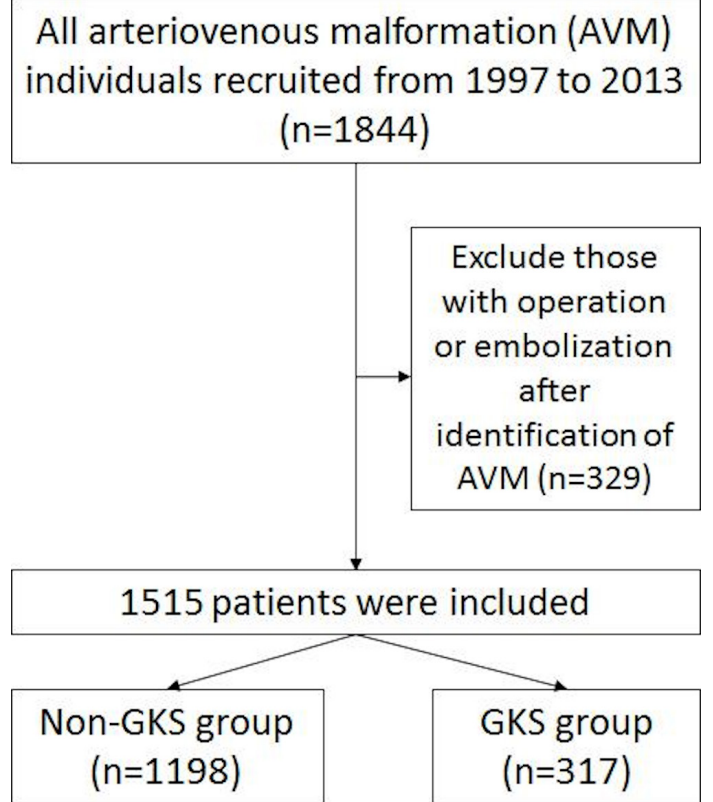

Figure 1 The flow chart of the cohort in this study. GKS, gamma knife radiosurgery.

medical records stored within the central bureau of Taiwan National Health Insurance Institute. These data informed the allocation of patients to either of the two treatment groups: GKS ( $\mathrm{n}=317)$ and non-GKS $(\mathrm{n}=1198)$. Figure 1 shows the study flow chart. The index date for each subject was the first date of diagnosis of AVM. All patients were followed up until spontaneous intracerebral haemorrhage (ICD-9-CM codes 431, and 432.9) or death, or the end of 2013. The age, sex and haemorrhage occurrence were obtained from medical records for statistical analysis.

\section{GKS technique}

All patients in the GKS group were treated with the Leksell gamma knife. All GKS was delivered via a multidisciplinary approach: the team consisted of a neurosurgeon, neuroradiologist, radiation oncologist and a medical physicist. The GKS technique followed a treatment guideline developed by the Taiwan Neurosurgical Society. The dosage prescription and number of lesions treated were individually determined by the expertise teams.

\section{Statistical analysis}

Distributions of the two groups according to age, sex and clinical characteristics were examined using $\chi^{2}$ tests. For estimating the risk of haemorrhage in patients of the different groups, we performed survival analysis using the Kaplan-Meier method, with significance based on the log-rank test. Multivariate Cox proportional hazards regression models were used to explore the relationship between treatment modality and haemorrhage, adjusted for age and sex. The crude and adjusted HR (aHRs) with $95 \%$ CIs were calculated. The proportional hazards assumption was tested graphically and by including the interaction of time with each covariate. A two-tailed $p$ 
Table 1 Comparison of demographic characteristics between non-GKS and GKS groups

\begin{tabular}{cllr}
\hline & $\begin{array}{l}\text { Non-GKS } \\
\text { group } \\
\text { (n=1198) }\end{array}$ & $\begin{array}{l}\text { GKS group } \\
\text { (n=317) }\end{array}$ & P value \\
\hline Age, mean \pm SD & $37.40 \pm 17.62$ & $33.08 \pm 15.48$ & $<0.001$ \\
\hline Age $<20, \mathrm{n}(\%)$ & $187(15.61)$ & $59(18.61)$ & 0.004 \\
$20 \leq$ age $\leq 40, \mathrm{n}(\%)$ & $515(42.99)$ & $159(50.16)$ & \\
Age $>40, \mathrm{n}(\%)$ & $496(41.10)$ & $99(31.23)$ & \\
Sex (male) (\%) & $665(55.51)$ & $169(53.31)$ & 0.484 \\
$\begin{array}{l}\text { Bleeding history (\%) } \\
\text { (Ruptured AVM) }\end{array}$ & & & \\
\hline
\end{tabular}

AVM, arteriovenous malformation; GKS, gamma knife radiosurgery.

$<0.05$ was considered statistically significant. All statistical analyses were performed with SAS (V.9.2; SAS Institute).

Patient and public involvement

Patients or the public were not involved in the design and the conduct of the study.
RESULTS

Of the total 1515 patients analysed in the present study, 317 underwent GKS (GKS group), and 1198 were treated conservatively (non-GKS group). Table 1 presents the demographic and clinical characteristics of the two groups. Patients in the GKS group (mean \pm SD, $33.08 \pm 15.48$ years of age) tended to be younger than those in the nonGKS group $(37.40 \pm 17.62$ year of age $)(p<0.001)$. Of all the AVM patients, the more patients in GKS group have prior bleeding history as compared with non-GKS group ( $59.66 \%$ vs $45.66 \%$, respectively, $\mathrm{p}<0.001)$. No significant differences in sex were identified between the two groups.

During the follow-up period of 15 years, all AVM patients in GKS group had lower incidence of bleeding than non-GKS group (aHR 0.61; 95\% CI 0.40 to 0.92) (table 2). The bleeding risk of ruptured AVMs was significantly lower in GKS group than in the non-GKS group (aHR $0.34 ; 95 \%$ CI 0.19 to 0.62 ). On the other hand, the bleeding risk of unruptured AVMs was significantly higher in the GKS group than in the non-GKS group (aHR 1.95; 95\% CI 1.04 to 3.65) (table 2). To elucidate the relationship between age and bleeding risk in patients with AVMs, patients were stratified into three subgroups according to

Table 2 Comparison of haemorrhage rates (per 100 patient-years) between non-GKS group and GKS group in AVM patients were stratified by bleeding history (ruptured or non-ruptured AVMs)

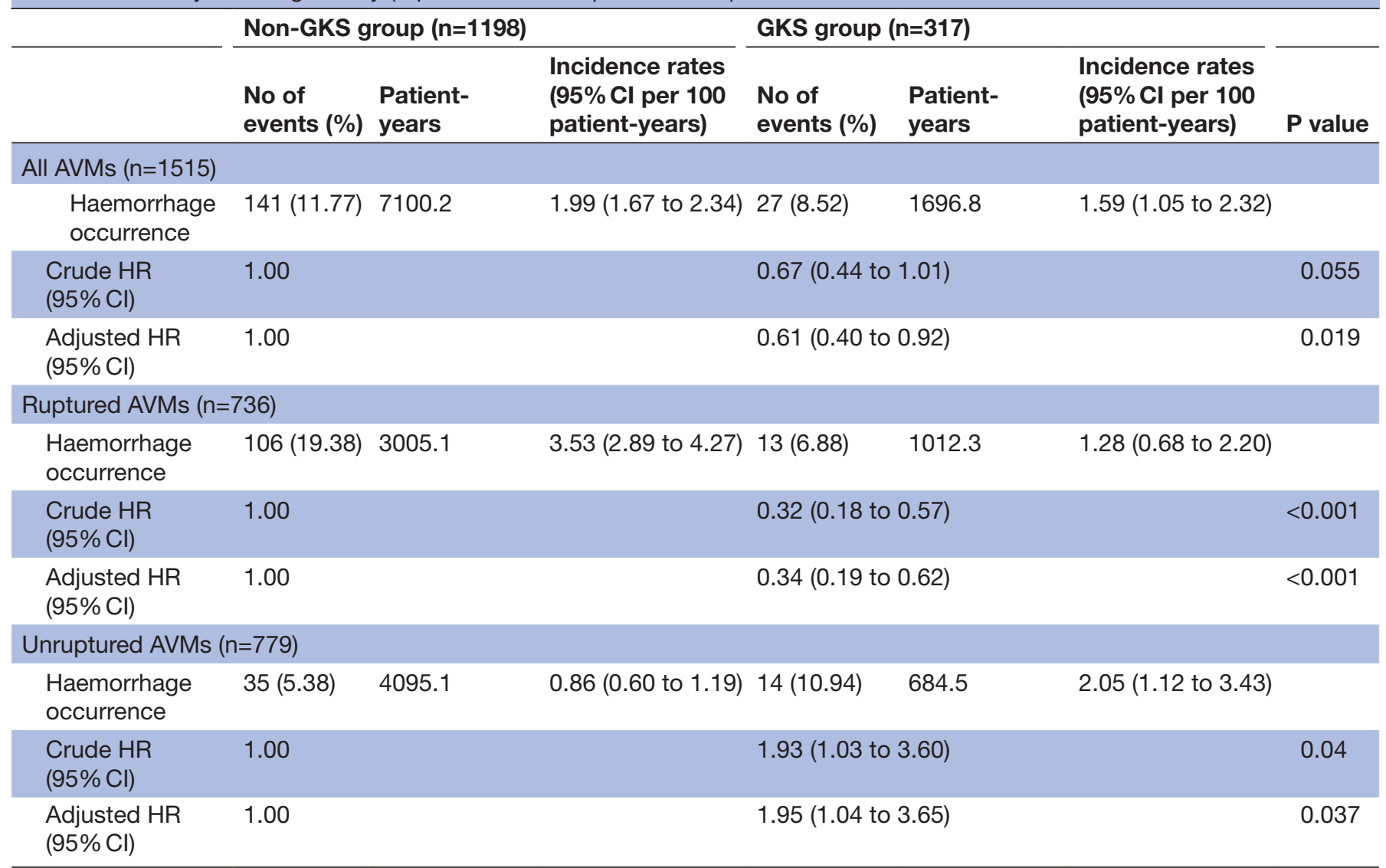

Adjusted for age and sex.

AVM, arteriovenous malformation; GKS, gamma knife radiosurgery. 

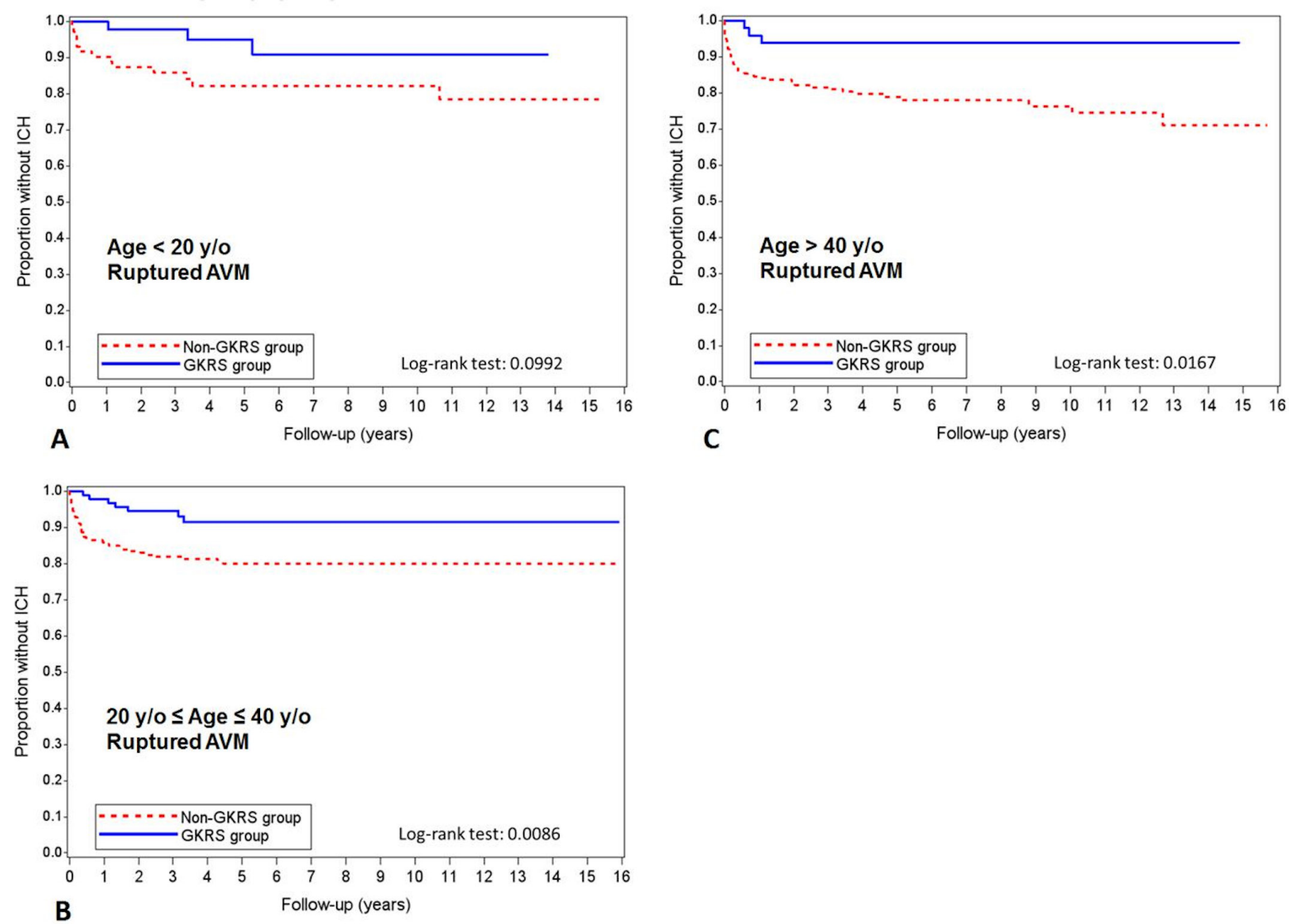

Figure 2 Kaplan-Meier curves showing cumulative rates of haemorrhage in ruptured AVM patients in relation to treatment modalities (non-GKS group vs GKS group) and age: (A) age <20, (B) age between 20 and 40, (C) age >40 years. AVM, arterovenous malformation; GKS, gamma knife radiosurgery; $\mathrm{ICH}$, intrcerebralhaemorrhage.

age: $<20,20-40,>40$ years. In the patients with ruptured AVMs (figure 2), the incidence of bleeding was significantly lower in the 20-40 group (aHR 0.36 ; $95 \%$ CI 0.16 to 0.80 ) and the $>40$ group (aHR $0.27 ; 95 \%$ CI 0.08 to 0.85 ) (table 3). In the patients with unruptured AVMs (figure 3), the incidence of bleeding was significantly higher in the $>40$ group (aHR 3.21; $95 \%$ CI 1.12 to 9.14 ) (table 3).

Regardless of sex, patients with ruptured AVMs were associated with a significantly lower risk of bleeding if they were treated with GKS (women: aHR $0.33 ; 95 \%$ CI 0.14 to 0.78 ; men: aHR 0.36 ; $95 \%$ CI 0.16 to 0.79 ) (table 3 ). The relationship between bleeding risk and sex was significantly stronger in GKS group compared with non-GKS group among men with unruptured AVMs (aHR 2.36; $95 \%$ CI 1.10 to 5.03).

\section{DISCUSSION}

In patients with untreated AVMs, the annual risk of haemorrhage is approximately $2 \%-4 \% .{ }^{10}$ The major goal in the management of AVM is the elimination of haemorrhage risk, which can be achieved through microsurgical resection, endovascular embolisation, radiosurgery or a combination of these procedures. Although surgical resection remains the principal means by which to obtain early obliteration of AVMs, GKS has been widely accepted as an effective alternative to surgery or embolisation for patients with smaller AVMs or those deeply situated in eloquent areas. ${ }^{11}$

This is the first nationwide Asian population-based study that considered the effect of GKS on AVMs with a follow-up of 15 years. According to the statistics of the Taiwanese NHI programme, approximately $97.11 \%$ of Taiwanese citizen (2002: 21, 869, 478 of 22, 520, 776) are enrolled in the NHI. ${ }^{9}$ According to the regulations of the NHI, patients receive a certificate of "catastrophic illness' when first diagnosed with cerebral AVMs. Within the certificate's validity period, patients are not required to pay any copayments for outpatient or inpatient care. Certifications of catastrophic illness are approved by the members in a specific review committee of the NHI. The ICD-9 code and certificate of catastrophic illness together confirmed the diagnoses of AVM for all patients enrolled in this study.

According to our database, significantly more patients with ruptured AVMs underwent GKS than patients with unruptured AVMs (59.62\% and $45.66 \%$, respectively) (table 1). Overall, the annual haemorrhage rate of AVMs 


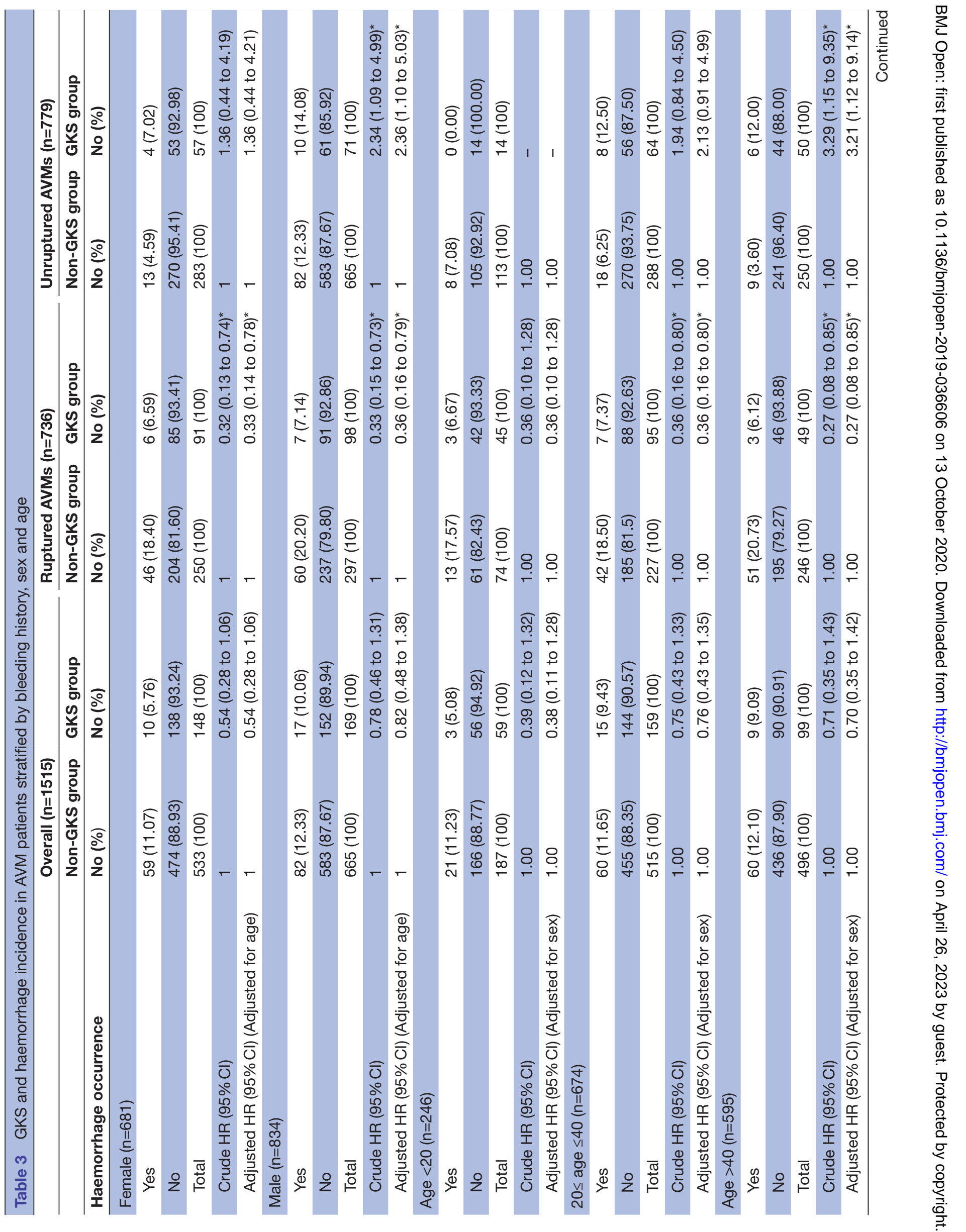


was lower in the GKS group (1.59 per 100 patient-years) than the non-GKS group (1.99 per 100 patient-years). Among the patients with ruptured AVMs, the annual risk of bleeding in the GKS group (1.28 per 100 patientyears) was significantly lower than that in the non-GKS group (3.53 per 100 patient-years). Our results indicate that ruptured AVMs require aggressive treatment to prevent subsequent haemorrhage, which occurs at a higher rate after the initial haemorrhage. The metaanalysis conducted by Gross $e t a l^{10}$ reported that unruptured AVMs had a $2.2 \%$ overall annual risk of bleeding and that ruptured AVMs had a $4.5 \%$ overall annual risk of bleeding rate. Compared with our national data, those values of previous reports were higher than ours might indicate possible overestimation of the annual bleeding rates due to patient selection bias and disparate patient referral systems in the different countries.

The management of unruptured AVMs is controversial and multifactorial, and the risks of treatment must be outweighed by those of the natural history of the AVM. ARUBA and the Scottish Audit of Intracranial Vascular Malformations prospective, comparative AVM cohort study indicate that patients with unruptured AVMs who received conservative management had better outcomes than those who underwent intervention. ${ }^{812}$ The present study showed GKS increased haemorrhage risk in unruptured AVM patients above age of 40. Our results seemed to be consistent with the results of ARUBA study. In ARUBA study, of the 223 patients with data available, the mean age of enrollees was $44.5 \pm 12.3$ years $($ mean $\pm \mathrm{SD}){ }^{7}$ Compared with our patients, the mean age of non-GKS group is $37.4 \pm 17.6$ years and GKS group is $33.1 \pm 15.5$ years, the patients of ARUBA on average are older than our patients. The older patients with unruptured AVM after GKS may have higher haemorrhage risk. Further we had longer followed up period of 15 years to observe the natural course of AVM compared with ARUBA study with 33 months.

However, the recent trials conducted by Tonetti et $a l,{ }^{13}$ which included 233 ARUBA-eligible patients with unruptured AVM patients who received follow-ups for a mean of 8.4 years, the administration of radiosurgery was associated with significantly lower annual rates of haemorrhage $(0.4 \%)$ and stroke or death $(0.8 \%)$ during the subsequent 3 years than conservative management. Ding $e t a l^{14}$ performed a retrospective cohort international study with 938 patients with unruptured AVMs who received follow-ups for a median of 7 years and reported favourable outcomes in $61 \%$ of patients who received radiosurgery, with an annual postradiosurgery haemorrhage rate of $1.4 \%$. In our database, the annual bleeding risk of unruptured AVM was significantly higher in the GKS group $(2.05 \%)$ than in the non-GKS group $(0.86 \%)$. When the analysis of patients with unruptured AVMs was performed according to age, no significant differences in annual bleeding risk were found between the patients of $<20$ years and those between 20 and 40 years old; the bleeding risk was significantly higher in patients aged 

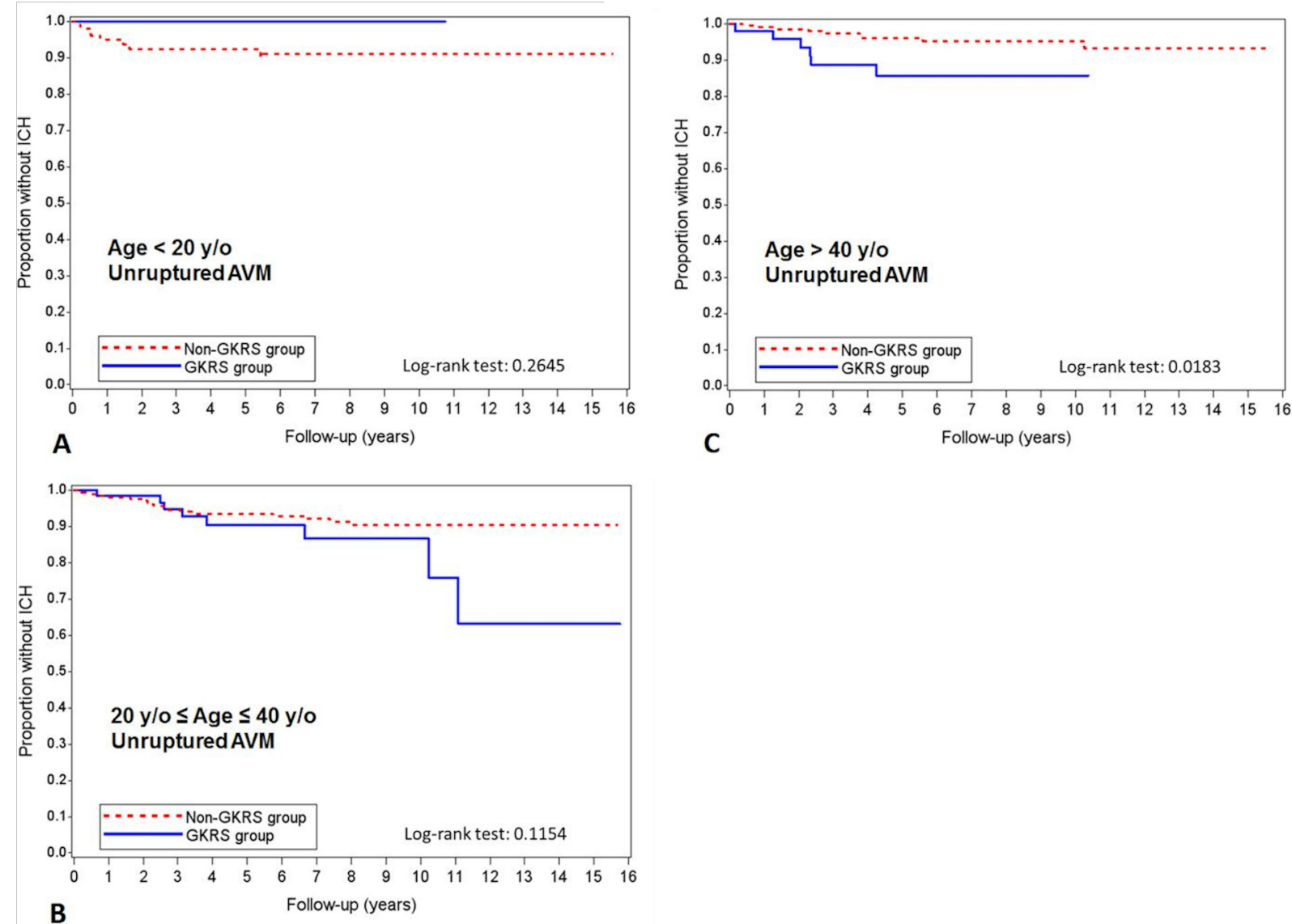

Figure 3 Kaplan-Meier curves showing cumulative rates of haemorrhage in unruptured AVM patients in relation to treatment modalities (non-GKS group vs GKS group) and age: (A) age <20, (B) age between 20 and 40, (C) age >40 years. AVM, arterovenous malformation; GKS, gamma knife radiosurgery; ICH, intrcerebralhaemorrhage.

$>40$ years (table 3 ). Tonetti et $a l^{13}$ performed an ARUBAeligible trial with 233 patients who had unruptured AVMs and received follow-ups for a median of 8.9 years and found that patients younger than 40 years had a significantly lower overall rate of experiencing stroke or death after receiving radiosurgery ( $7 \%$ vs $20 \%$ ).

Tong et al performed a retrospective cohort study that included a total of 3299 AVMs and found that bleeding frequency was highest in young patients: $<10(72.7 \%), 10-19$ $(67.4 \%)$ and $20-29(59.8 \%)$ years of age. Through our population-based study, we observed the following bleeding risks among patients with unruptured AVMs who did not receive GKS: $<20(7.08 \%), 20-40(6.25 \%),>40$ years of age $(3.6 \%)$. These values were lower than those reported previously. However, the haemorrhage rate of patients with unruptured AVMs in the GKS group increased significantly when they exceed 40 years of age (12\%). The haemodynamics of older patients with unruptured AVMs could be well matured and stabilised, the radiosurgery-mediated changes in vascular structure and haemodynamic status may induce bleeding. Yang et al helped to elucidate the pathophysiology underlying the increased rate of postradiosurgery haemorrhage in the patients who received pretreatment for venous stenosis. ${ }^{15}$ The identified risk factors included the following: advanced age, female sex, the presence of venous stenosis, large AVM volumes and previous haemorrhage. ${ }^{15}$ In the present study, we found that bleeding risk gradually decreases with ageing. This finding might imply that the older the patient, the more stable the brain haemodynamics in cases of unruptured AVMs. Tong et al $t^{4}$ reported that age has a significant effect on the clinical presentation of AVMs at the time of initial diagnosis. The haemorrhage rate was highest in patients younger than 30 years of age and extremely high in children younger than 10 years of age. AVMs presenting in children are thus a more malignant type of AVM that is more likely to haemorrhage. ${ }^{16}$ Biological differences in brain haemodynamics may exist between AVMs presenting in children and those presenting in adults. The maturity of AVMs and the stabilisation of AVM haemodynamics in adult as they grow probably accounts for the lower bleeding risk observed in older patients. However, Stapf $e t a l^{17}$ reported the highest bleeding frequencies among patients of $>60$ years of age. The discrepancies between our study and those previous may also be the result of the different geographic origins of the patients and variances in institutional referral patterns.

\section{Limitation}

The present study is retrospective and population based with limited clinical information. Information regarding detailed clinical and functional outcomes during the 
period of follow-up was unavailable. In addition, the grading of AVMs and neurological severity of the haemorrhage could not be ascertained, limiting our insight into the significance of the effect of GKS on AVMs of different grades. Furthermore, we lacked detailed data concerning radiosurgery dose planning, as it varied extensively across centres. Therefore, we were unable to assess the relative efficacy and safety profiles of radiosurgery in a comparable cohort of patients who were conservatively managed.

\section{CONCLUSIONS}

Our results suggest that GKS provided an acceptable risk and benefit profile in reducing the subsequent risk of haemorrhage in patients with ruptured AVMs. However, whether unruptured AVMs should be treated and what treatment approach is the most effective remain the subjects for debate. For appropriately selected unruptured AVMs, GKS provides a minimally invasive procedure that appears to achieve a reasonable outcome with low procedure-related morbidity. For older adults with unruptured AVMs, the present study indicates that GKS might increase the risk of subsequent bleeding. In patients with unruptured AVMs, the benefits and risks of radiosurgical intervention can only be determined with long-term follow-up.

Acknowledgements The authors thank the Center for Database Research of E-DA Healthcare Group for statistical assistance.

Contributors Guarantor of integrity of the entire study: C-LC, H-JC and C-LL. Study concepts and design: C-LC and C-LL. Literature research: K-WW and S-AY. Data analysis: C-LC, K-WW, S-AY, JTT and C-LL. Statistical analysis: C-LC and C-LL. Manuscript preparation and editing: C-LC, JTT, H-JC and C-LL.

Funding This work was financially supported by the Center for Database Research, E-DA Healthcare Group (EDAD 10605).

Competing interests None declared.

Patient consent for publication Not required.

Ethics approval This study was approved by the institutional review board of E-DA hospital (EMRP-108-048).

Provenance and peer review Not commissioned; externally peer reviewed.

Data availability statement Data may be obtained from a third party and are not publicly available. Data may be obtained from the Center for Database Research, E-DA Healthcare Group and are not publicly available.

Open access This is an open access article distributed in accordance with the Creative Commons Attribution Non Commercial (CC BY-NC 4.0) license, which permits others to distribute, remix, adapt, build upon this work non-commercially, and license their derivative works on different terms, provided the original work is properly cited, appropriate credit is given, any changes made indicated, and the use is non-commercial. See: http://creativecommons.org/licenses/by-nc/4.0/.

\section{ORCID iD}

Cheng-Loong Liang http://orcid.org/0000-0002-6747-0053

\section{REFERENCES}

1 Al-Shahi R, Warlow C. A systematic review of the frequency and prognosis of arteriovenous malformations of the brain in adults. Brain 2001;124:1900-26.

2 Hofmeister C, Stapf C, Hartmann A, et al. Demographic, morphological, and clinical characteristics of 1289 patients with brain arteriovenous malformation. Stroke 2000;31:1307-10.

3 Stapf C, Mast H, Sciacca RR, et al. Predictors of hemorrhage in patients with untreated brain arteriovenous malformation. Neurology 2006;66:1350-5.

4 Tong X, Wu J, Lin F, et al. The effect of age, sex, and lesion location on initial presentation in patients with brain arteriovenous malformations. World Neurosurg 2016;87:598-606.

5 Starke RM, Kano H, Ding D, et al. Stereotactic radiosurgery for cerebral arteriovenous malformations: evaluation of long-term outcomes in a multicenter cohort. J Neurosurg 2017;126:36-44.

6 Maruyama K, Kawahara N, Shin M, et al. The risk of hemorrhage after radiosurgery for cerebral arteriovenous malformations. $N$ Engl $J$ Med 2005;352:146-53.

7 Mohr JP, Parides MK, Stapf C, et al. Medical management with or without Interventional therapy for unruptured brain arteriovenous malformations (ARUBA): a multicentre, non-blinded, randomised trial. Lancet 2014;383:614-21.

8 Link TW, Winston G, Schwarz JT, et al. Treatment of unruptured brain arteriovenous malformations: a single-center experience of 86 patients and a critique of the a randomized trial of unruptured brain arteriovenous malformations (ARUBA) trial. World Neurosurg 2018;120:e1156-62.

9 Liang C-L, Liliang P-C, Chen T-B, et al. The risk of cataractogenesis after gamma knife radiosurgery: a nationwide population based case-control study. BMC Ophthalmol 2017;17:40.

10 Gross BA, Du R. Natural history of cerebral arteriovenous malformations: a meta-analysis. J Neurosurg 2013;118:437-43.

11 Starke RM, Yen C-P, Ding D, et al. A practical grading scale for predicting outcome after radiosurgery for arteriovenous malformations: analysis of 1012 treated patients. J Neurosurg 2013;119:981-7

12 Al-Shahi Salman R, White PM, Counsell CE, et al. Outcome after conservative management or intervention for unruptured brain arteriovenous malformations. JAMA 2014;311:1661-9.

13 Tonetti DA, Gross BA, Atcheson KM, et al. The benefit of radiosurgery for ARUBA-eligible arteriovenous malformations: a practical analysis over an appropriate follow-up period. J Neurosurg 2018;128:1850-4.

14 Ding D, Starke RM, Kano H, et al. Radiosurgery for unruptured brain arteriovenous malformations: an international multicenter retrospective cohort study. Neurosurgery 2017;80:888-98.

15 Yang W, Luksik AS, Jiang B, et al. Venous stenosis and hemorrhage after radiosurgery for cerebral arteriovenous malformations. World Neurosurg 2019;122:e1615-25.

16 Fullerton HJ, Achrol AS, Johnston SC, et al. Long-Term hemorrhage risk in children versus adults with brain arteriovenous malformations. Stroke 2005;36:2099-104.

17 Stapf C, Khaw AV, Sciacca RR, et al. Effect of age on clinical and morphological characteristics in patients with brain arteriovenous malformation. Stroke 2003;34:2664-9. 